\title{
TouchIt3D: Technology (not only) for Tactile Maps
}

\author{
Radek Barvir, Alena Vondrakova, Jan Brus* \\ ${ }^{a}$ Department of Geoinformatics, Faculty of Science, Palacký University Olomouc, 17. listopadu 50, Olomouc, Czechia \\ radek.barvir@upol.cz,jan.brus@upol.cz,alena.vondrakova@upol.cz. \\ * Corresponding author
}

Keywords: 3D printing, TouchIt3D, Tactile Maps, Technology

\begin{abstract}
:
The majority of information has a spatial context that can be represented on the map, while maps are presenting the real world in the simplified and generalised way, focusing on the key features or specific topic. For some kinds of users, the map as the representation of the real spatial context is not only the possibility but also the necessity. Among these people belong people with visual impairments.
\end{abstract}

The number of visually impaired people increases every year and to their full-fledged integration into society is devoted considerable attention. But People with visual impairments are the target group with specific user needs, and the conventional map is insufficient for them. Along the growing number of visually impaired people importance of tactile cartography is increasing.

Currently, there are many technologies used for creating tactile maps, including very primitive and cheap solutions as well as advanced methods. The simplest way is drawing on the hand which brings only the real-time perception which needs to memorise for next uses. Another technique of hand embroidery consists of thick fibre placed on the cardboard or different paper type. More accurate is drawing on a special paper for blind or using dense colour gels. Also, some kinds of machinery producing technologies are used, e. g: shaping carton, plastic or metal. Braille printers can produce not very complicated tactile maps using 3D dots. Similar results can be obtained using serigraphy. Very popular is printing on heat-sensitive paper as mentioned before in the case of haptic maps by Mapy.cz. Another possibility is to use rubberized colours and nowadays popular technology of 3D printing (Vozenilek and Ludikova, 2010).

At the Department of Geoinformatics, Faculty of Science, Palacký University Olomouc, Czechia, the research team developed prototypes and methodology for the creation of the modern type of 3D tactile maps, linkable with mobile devices (Barvir et al., 2018). Interactive tactile maps connectable with mobile devices bring new opportunities to develop tactile map production. The prototypes have been verified in practice in cooperation with educational centres for people with visual impairment and blind people, and special schools. It is comprehensive research focusing a lot of scientific challenges. The contribution would like to summarise the most significant findings of the research.

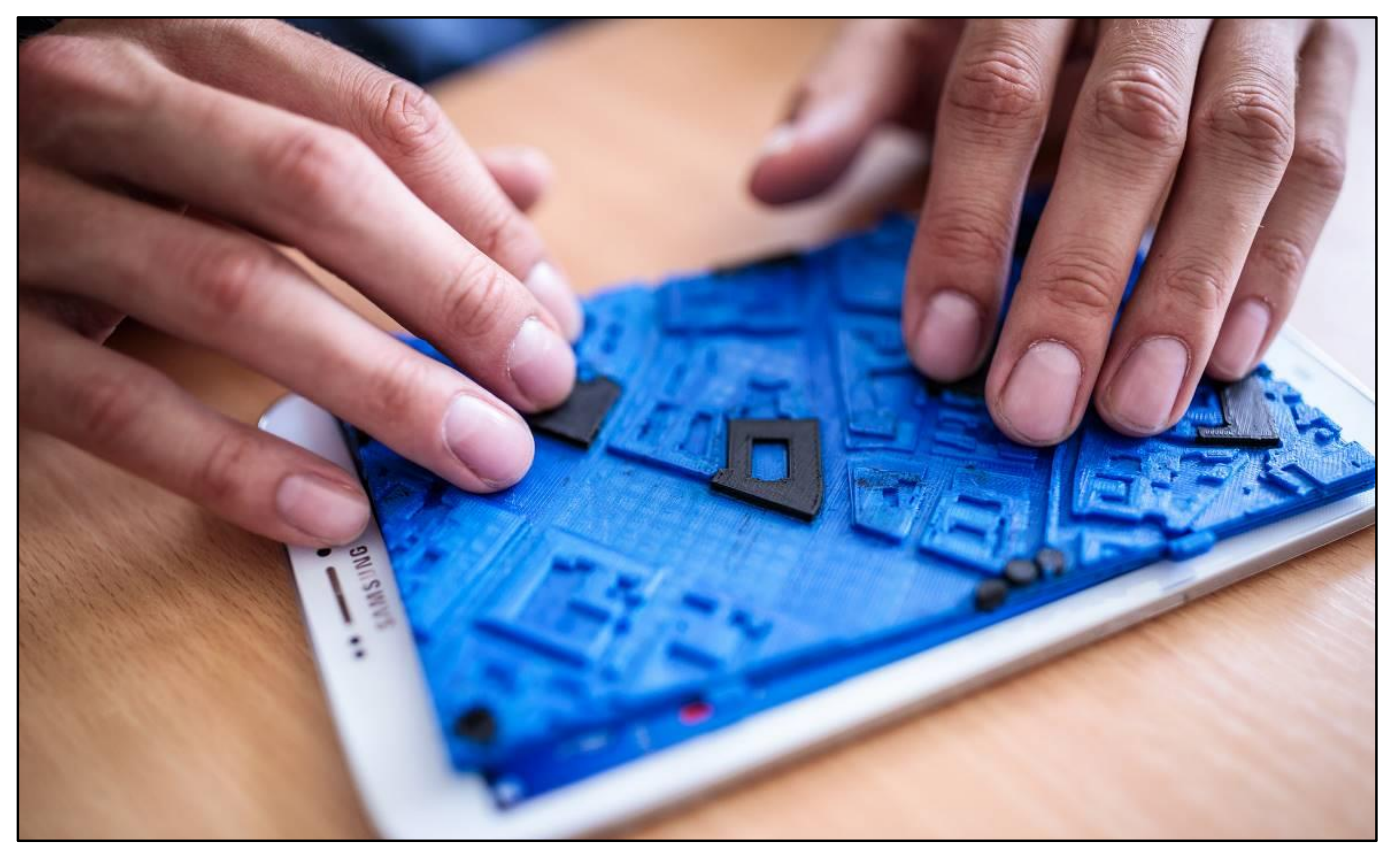

Figure 1. Modern type of 3D tactile map developed at the Department of Geoinformatics, Faculty of Science, Palacký University Olomouc, using the smart phone for the audio-module part of the tactile map, used by the people with visual impairment. 
The developed TouchIt3D technology is based on linking 3D objects, such as tactile maps, 3D models, controls, etc., with a mobile tablet or mobile phone using a combination of conductive and non-conductive filament. Each model is linked to an individual mobile application layout that initiates a pre-action based on user suggestions done within touching the model. For example, such an action may be a vibration or a speech command when the person with visual impairment touch inappropriate map symbol. As example can be introduced a listing of current public transport departures after the user touches the bus-stop map symbol on the 3D transport terminal plan. Data can be acquired in real time via Internet as the tablet can be connected to $\mathrm{WiFi}$ or cellular network. TouchIt3D technology is primarily focused on the presentation of spatial data and navigation for the public, people with visual or other impairment.

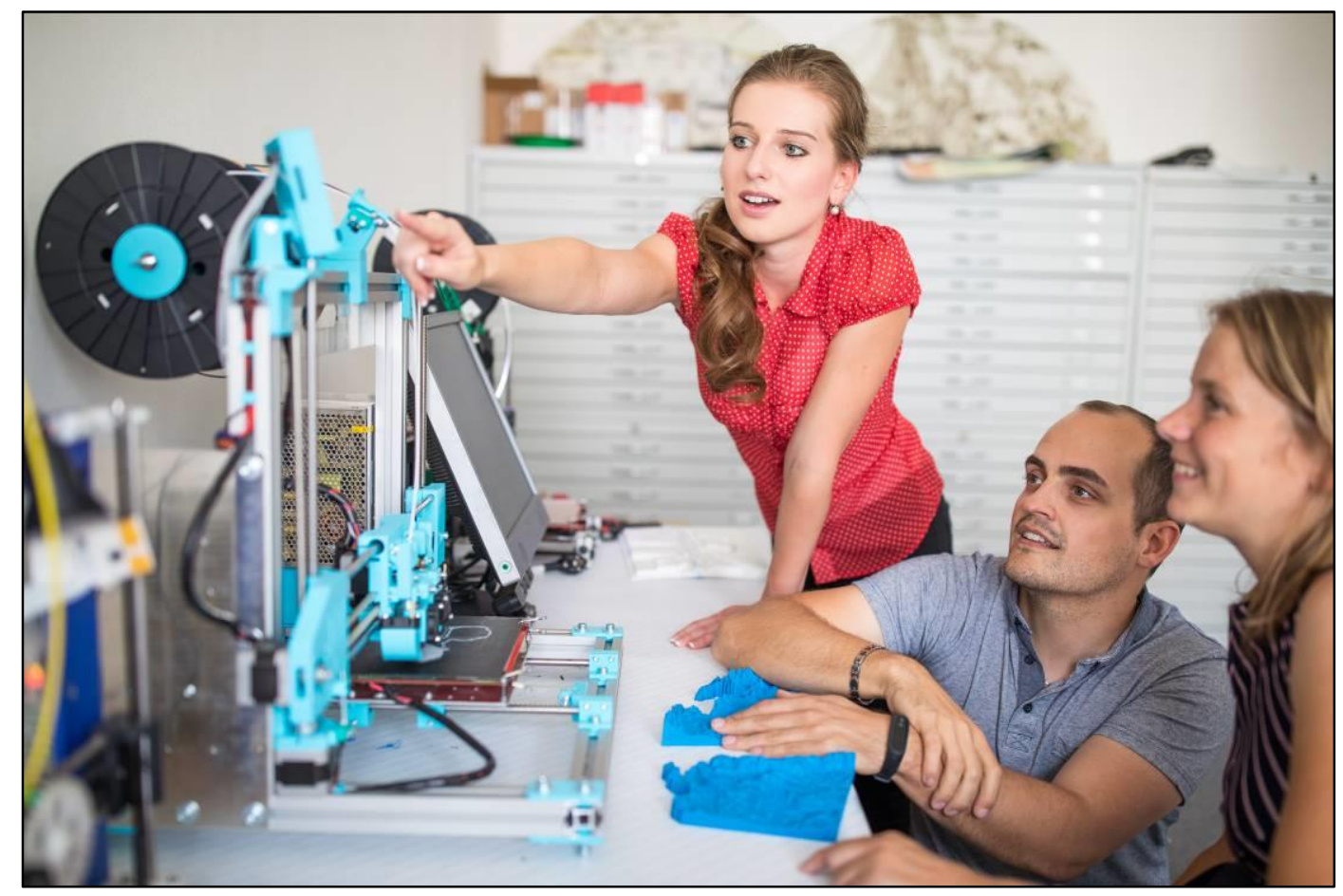

Figure 2. Modern type of low-cost 3D printers at the Department of Geoinformatics, Faculty of Science, Palacký University Olomouc. One tactile map (size 20 to $13 \mathrm{~cm}$ ) is printed within few hours according to the required detail printing (resolution).

There are two ways how to create such tactile map. The first way is to prepare all the data manually. Another approach is the semi-automatic workflow. This approach is significantly different from previous workflows of producing maps for people with visual impairment. The solution based on the open-source and free software and data together with sharing electronic part of the map in the form of tablet dramatically lowered costs of tactile maps production. The designed scripts and models also reduced the time necessary to spend by map designing up to a minimum. User testing provided all data required for the improvement, and maximal adaptation of the cartographic visualisation methods to the target user needs. Nevertheless, maps partly automatically done and based on crowdsourcing data cannot bring the same quality as individually made tactile maps.

The main aim of the research is to find a workflow of interactive tactile maps creation using the TouchIt3D technology. The research also deals with setting appropriate parameters of the map, e.g. the map scale, cartographic symbol size, map content etc. This optimisation is done to fit the needs of people with visual impairment as much as possible on the one hand and taking into account the limitations of the map creation possibilities.

This research is implemented within the project Development of independent movement through tactile-auditory aids, Nr. TL01000507, supported by the Technology Agency of the Czech Republic. 